\title{
Thermal Effects on Atomic Friction
}

\author{
Yi Sang, Martin Dubé, and Martin Grant \\ Centre for the Physics of Materials, Physics Department, Rutherford Building, McGill University, \\ 3600 rue University, Montréal, Québec, H3A 2 T8 Canada
}

(Received 18 June 2001; published 8 October 2001)

\begin{abstract}
We model friction acting on the tip of an atomic force microscope as it is dragged across a surface at nonzero temperatures. We find that stick-slip motion occurs and that the average frictional force follows $|\ln v|^{2 / 3}$, where $v$ is the tip velocity. This compares well to recent experimental work, permitting the quantitative extraction of all microscopic parameters. We calculate the scaled form of the average frictional force's dependence on both temperature and tip speed as well as the form of the friction-force distribution function.
\end{abstract}

DOI: $10.1103 /$ PhysRevLett.87.174301

The study of friction between two surfaces presents numerous theoretical and experimental challenges [1,2]. Two macroscopic surfaces interact through many asperities. These asperities are on an atomic length scale, so macroscopic friction is inextricably linked with microscopic properties. A friction force microscope, the tip of which is in essence a single asperity [3-5], provides an ideal way to probe atomic friction by removing the complication of multiple asperities. Under a constant load, the low-velocity motion of the tip on a surface exhibits stick-slip behavior as the moving tip hops over an atomically defined substrate [6-10]. In principle, such results can give a quantitative description of friction at the microscopic level. However, this requires a theoretical understanding of the behavior of the tip on the substrate. This is the purpose of this Letter.

Recent studies have argued that, due to thermal fluctuations, the lateral friction force $F$ has a logarithmic dependence on velocity [4,5] $F \propto$ const $+\ln v$ where $v$ is the velocity of the support. This corresponds to linear creep between two surfaces in contact when the force acting on a substrate produces a small constant potential bias [11]. But this is quite different from what occurs when a tip is dragged across the substrate: the potential bias is continuously ramped up as the support is moved. We will call this ramped creep and will show that, at constant temperature $T$ [12-17],

$$
F \propto \text { const }-T^{2 / 3}|\ln v / T|^{2 / 3} .
$$

It is straightforward to discriminate between linear and ramped creep. Consider Figs. 1a and 1b, where the combined potential of the atomic substrate and the elastic tip is shown. Locally, it is sufficient to consider two wells. In that case, a useful analogy to (mean-field) nucleation at a first-order phase transition occurs $[12,13]$. When the bias is infinitesimally small, the energy, up to an additive constant, is well described locally by $E=F x+x^{2}-x^{4}$, where $F$ is friction and $x$ is displacement. Hence the barrier height is $\Delta E \sim$ (const $-F$ ), as for mean-field nucleation near the coexistence curve. In the steady state, the rate of thermal fluctuations is proportional to the veloc-
PACS numbers: 46.55.+d, 07.79.-v, 64.60.My, 81.40.Pq

ity. Since the rate is itself proportional to the probability of a fluctuation over this barrier, one obtains linear creep: the force depends logarithmically on the velocity. This is, however, an unlikely regime because the barrier is so high (see Fig. 1a). In contrast, as the tip is dragged over the substrate and the bias is ramped up, barrier-hopping fluctuations occur preferentially when the tip is close to slipping at the top of the barrier. At this point, the local combined potential can be written $E=F x-x^{3}$, where the origin of the $x$ axis has been conveniently shifted, and the second well has been replaced by an absorbing boundary condition (see Fig. 1b). Then we have $\Delta E \sim(\text { const }-F)^{3 / 2}$ and Eq. (1) for ramped creep is obtained. This is similar to mean-field nucleation near the spinodal $[12,13]$, and is calculated, as well as observed experimentally, for, e.g., magnetic-flux fluctuations in superconducting quantum interference devices [14-16], and in other contexts [17]. We give a detailed exposition of the algebra below. First, however, we solve a model numerically and show that the experimental regime corresponds to our physical picture.

The system composed of the tip of the force microscope interacting with a surface can be described by a modified Tomlinson model [7-10]. We thus consider a tip with longitudinal and transverse coordinate positions $x$ and $z$ and effective mass $M$. The tip moves under the influence of the surface adiabatic potential $E(x, z)$, coupled elastically to the support of the microscope, of coordinate $R(t)$ and $Z(t)$. For constant load, the distance $Z(t)-z$ is fixed, so the transverse coordinate plays no role, and $R=v t$ with $v$ the constant speed at which the tip is dragged. Including the random effect of substrate fluctuations, we have the Langevin equation

$$
M \frac{d^{2} x}{d t^{2}}+M \gamma \frac{d x}{d t}+\frac{\partial E(R, x)}{\partial x}=\xi(t),
$$

where $M$ is the mass of the tip, $\gamma$ is the microscopic friction coefficient, $E$ is the combined surface-tip potential, and $\xi$ is a random noise satisfying the fluctuation-dissipation relation $\left\langle\xi(t) \xi\left(t^{\prime}\right)\right\rangle=2 M \gamma k_{B} T \delta\left(t-t^{\prime}\right)$, where the angular brackets denote an average, and $k_{B}$ is Boltzmann's constant. The combined surface-tip potential has the form 
(a)

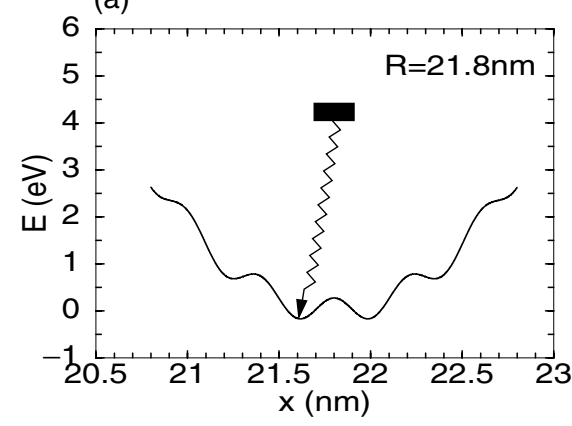

(c)

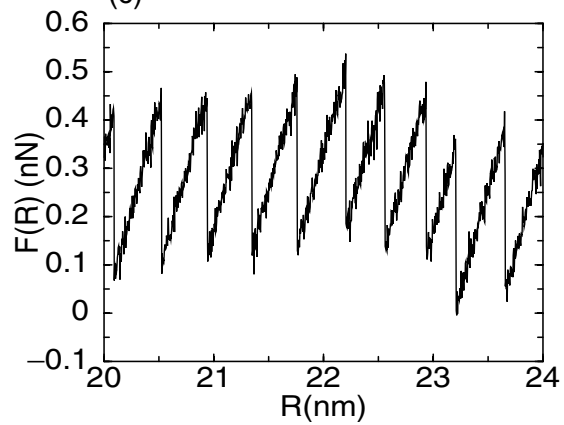

(b)

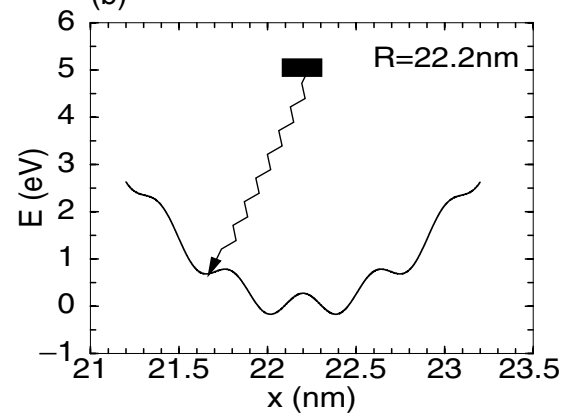

(d)

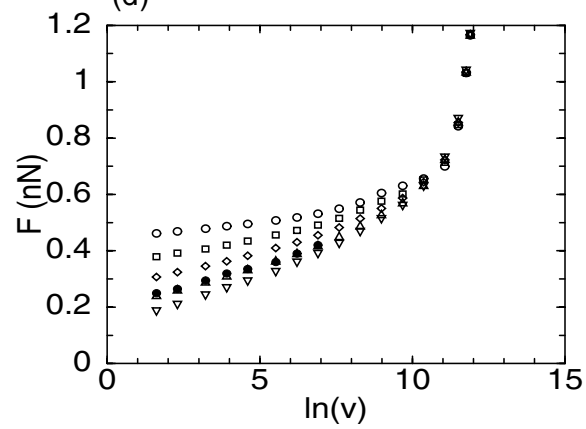

FIG. 1. (a) Schematic representation of the combined tip-surface potential just after a slip event. At this point, the energy barrier is high and thermally activated transitions improbable. (b) As the support is moved, the energy barrier diminishes and transitions become more likely. Inset (c) shows typical stick-slip behavior of the instantaneous friction force as the support is moved at velocity $v=25 \mathrm{~nm} / \mathrm{sec}$ for a temperature $T=293 \mathrm{~K}$. Inset (d) shows the average friction force for different temperatures. Open circles, squares, diamonds, triangles up, and triangles down correspond, respectively, to temperatures $T=53,133,213,293$, and $373 \mathrm{~K}$; closed circles are the experimental data of Ref. [5]. The units of velocity $v$ are $\mathrm{nm} / \mathrm{sec}$.

$$
E(R, x)=\frac{k}{2}[R(t)-x]^{2}-U \cos \left(\frac{2 \pi x}{a}\right),
$$

where $U$ is the surface barrier potential height, $a$ is the lattice constant, and $k$ is the elastic constant of the tipsupport coupling. There are a series of potential wells $\tilde{x}(R)$ given by the solution to $\partial E(R, x) /\left.\partial x\right|_{\tilde{x}}=0$, with the instantaneous lateral friction force on the support $F(t)=$ $k[R(t)-x(t)]$. A typical sequence of the stick-slip motion of $F$ is shown in Fig. 1c.

The relevant time scales of the problem are $\gamma^{-1}$, the viscous time scale for dissipation of energy from the tip to the surface [18], $p_{0}=\sqrt{M a^{2} / U}$, the oscillation period of the mass in the surface potential, and the tip resonance period $p_{k}=\sqrt{M / k}$. These parameters are effective in the sense that their precise value may depend on the load applied to the friction force microscope [19]. The friction coefficient must be larger than the critical value needed to observe overdamped stick-slip behavior at zero temperature. Within the Tomlinson model, this value is $\sim 2 \omega_{k}[7,9,10]$. To first order in the deviations of the tip from an integer position, the instantaneous force can be approximated as $F(t) \simeq\left[k /\left(1+\Omega_{k}^{2}\right)\right] R(t) \equiv \tilde{k} R$, where $\Omega_{k}=p_{0} /\left(2 \pi p_{k}\right)$.

Equation (2) was simulated using Ermak's algorithm [20] with parameters consistent with experimental conditions. The lattice parameter $a=0.4 \mathrm{~nm}$ and the effec- tive spring constant $\tilde{k}=0.86 \mathrm{~N} / \mathrm{m}$ [21] were taken from the data of Ref. [5], obtained under constant load $F_{n}=$ $0.65 \mathrm{nN}$. The parameters $U=0.27 \mathrm{eV}$ and $\gamma=8.9 \times$ $10^{5} \mathrm{sec}^{-1}$ and spring resonance frequency $\left(2 \pi p_{k}\right)^{-1}=$ $52 \mathrm{MHz}$ were adjusted to obtain the best fit with the experimental data. The mass $M=8.7 \times 10^{-12} \mathrm{~kg}$ then follows. The tip oscillation frequency $2 \pi p_{0}^{-1}=1.1 \times 10^{6} \mathrm{sec}^{-1}$ is a comparatively small attempt frequency compared to usual values in adatoms' surface diffusion [22], which is why thermally activated events with very small barrier height are important.

The numerical simulations were performed over a large range of velocities $5 \mathrm{~nm} / \mathrm{sec} \leq v \leq 256 \mu \mathrm{m} / \mathrm{sec}$, with several temperatures $53 \leq T \leq 373 \mathrm{~K}$ and time steps $0.001 p_{0} \leq \delta t \leq 0.01 p_{0}$. From the stick-slip motion of $F$, we extracted the average lateral force as a function of velocity, for different temperatures. This is shown in Fig. 1d. To test the prediction for ramped creep, we first extracted the constant force in Eq. (1), $F_{c}$, from the data at different temperatures, but fixed ratio of the scaled velocity $v / T$, as shown in the top inset of Fig. 2 . With this constant removed, the prediction from Eq. (1) corresponds to a universal form, which is independent of temperature. Figure 2 shows a very good scaling collapse of the dependence of the average force on velocity for numerical data from five different temperatures, as well as for experimental data from the work of Gnecco et al. 


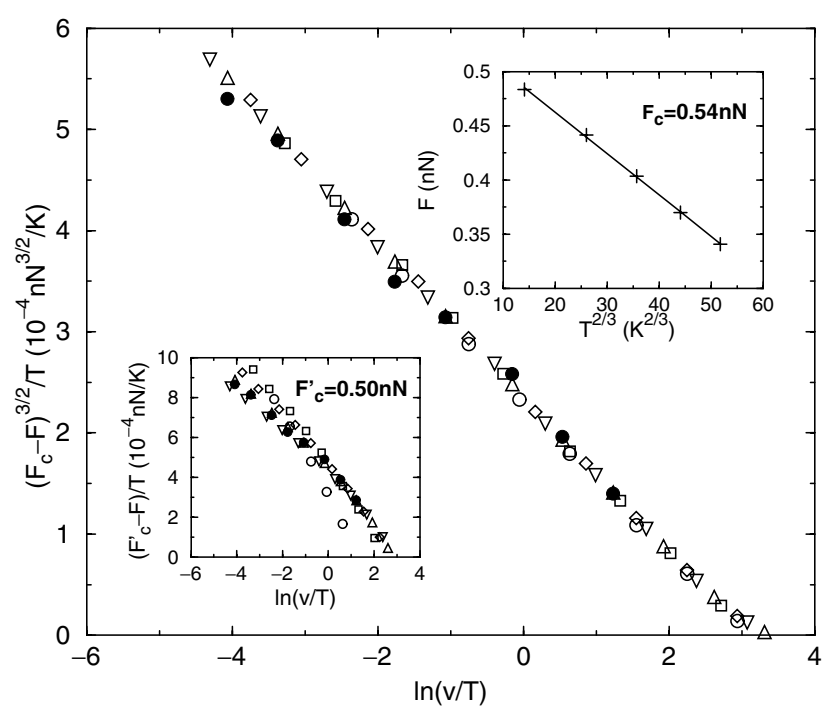

FIG. 2. Justification of the scaling form, Eq. (1). The upper inset shows the value $F_{c}$ as extracted from the data of the friction force shown in Fig. 1 for different temperatures between 53 and $373 \mathrm{~K}$ and fixed ratio $T / v=1 \mathrm{~K} /(\mathrm{nm} / \mathrm{sec})$. The lower inset shows significantly worse scaling for linear creep, using $\ln (v / T)$. The units of velocity $v$ are $\mathrm{nm} / \mathrm{sec}$ temperature is in degrees kelvin.

[5]. This collapse well confirms the prediction of ramped creep from Eq. (1). The small deviations from the logarithmic behavior at large values of $v / T>1$ indicate the approach to the zero-temperature stick-slip motion [7-9]. The bottom inset of Fig. 2 shows that, in contrast, linear creep does not give a good scaling collapse.

These results can be explained analytically by considering the thermal activation transition of the tip out of a single metastable well and the distribution of the support's position $P(R)$ when such a transition occurs [14,16]. Translational invariance allows us to concentrate on one such minimum, of value $\tilde{x}=0$ if $R=0$. As the support is moved, the barrier potential to the next minimum vanishes at some critical position $R_{c}$ [14], determined by $\partial E / \partial x_{c}=\partial^{2} E / \partial x_{c}^{2}=0$ :

$$
\frac{2 \pi R_{c}}{a}=\frac{2 \pi x_{c}}{a}+\frac{1}{\Omega_{k}^{2}} \sin \left(\frac{2 \pi x_{c}}{a}\right),
$$

where $2 \pi x_{c} / a=\cos ^{-1}\left(-\Omega_{k}^{2}\right)$. Note that the support's motion corresponds to a continuous linear ramping of the potential. Provided that the velocity of the support is slow enough, there can occur thermally activated transitions between two minima before the critical position $R_{c}$ is reached. This is described by the Kramer's rate [23]

$$
\tau^{-1}=\frac{\Omega^{2}}{2 \pi \gamma} e^{-\Delta E / k_{B} T},
$$

where $\Omega$ and $\Delta E$ correspond, respectively, to the instantaneous effective oscillation frequency and barrier height. Thermally activated transitions are most likely to occur when the support's position is close to the critical value. To lowest order in the bias $f(t) \equiv 1-\left[R(t) / R_{c}\right] \ll 1$, the minimum and maximum of the potential are easily obtained, and one finds [14]

$$
\Omega=\frac{2 \pi}{\sqrt{p_{0} p_{k}}}\left(\frac{4 \pi R_{c}}{a}\right)^{1 / 4}\left(1-\Omega_{k}^{4}\right)^{1 / 8} f^{1 / 4}
$$

and

$$
\Delta E=\frac{2}{3} U\left(\frac{4 \pi R_{c}}{a}\right)^{3 / 2} \frac{\Omega_{k}^{3}}{\left(1-\Omega_{k}^{4}\right)^{1 / 4}} f^{3 / 2} .
$$

Note the barrier height vanishes as $f^{3 / 2}$ as anticipated above. Likewise, the factor $f^{1 / 4}$ justifies the high-friction limit $(\gamma \gg \Omega$ ) of the prefactor in Eq. (5).

The transition rate represents a measure of the time needed before a thermally activated transition takes place. In particular, $W[R(t)]=\exp -\int_{t_{0}}^{t} \tau^{-1}\left[R\left(t^{\prime}\right)\right] d t^{\prime}$ gives the probability that a transition has not taken place at time $t$. Because of the exponential character of the transition rate, the probability for transition is much greater when $f \ll 1$ and insensitive to the initial support position. The distribution of the support's position at which a transition occurs is then conveniently expressed in terms of the reduced bias $f^{*}=\left(\Delta E / k_{B} T\right)^{2 / 3}$ as [14]

$$
P\left(f^{*}\right)=\frac{3}{2} \frac{f^{* 1 / 2}}{v^{*}} \exp \left[-f^{* 3 / 2}-\left(e^{-f^{* 3 / 2}}\right) / v^{*}\right],
$$

where the dimensionless velocity

$$
v^{*}(T, v)=2\left(\frac{v \gamma p_{0}^{2} U}{k_{B} T a}\right) \frac{\Omega_{k}^{2}}{\left(1-\Omega_{k}^{4}\right)^{1 / 2}}
$$

is essentially a function of the temperature, microscopic friction coefficient, and velocity of the support. The average of the distribution $\left\langle f^{*}\right\rangle$ is then found from Eq. (8), with value $\left\langle f^{*}\right\rangle=\left|\ln v^{*}\right|^{2 / 3}+\mathcal{O}\left(1 / \ln v^{*}\right)$ in the limit $v^{*} \ll 1$ $[14,16]$.

Once the average support's position at which a slip event occurs is known, it is straightforward to calculate the average lateral force as the integral of the instantaneous force over a cycle of the stick-slip motion [24]; the form of Eq. (1) then follows:

$$
F=F_{c}-\Delta F\left|\ln v^{*}\right|^{2 / 3},
$$

where the constants [25]

$$
F_{c}=\tilde{k}\left(R_{c}-\frac{a}{2}\right),
$$

and

$$
\Delta F=\frac{\pi U}{a}\left(\frac{3}{2} \frac{k_{B} T}{U}\right)^{2 / 3}\left(\frac{\left(1-\Omega_{k}^{4}\right)^{1 / 6}}{1+\Omega_{k}^{2}}\right) .
$$

With the parameters used in Fig. 2, Eqs. (9), (11), and (12) predict values of $F_{c}=0.55 \mathrm{nN}, \Delta F / T^{2 / 3}=1.91 \times$ $10^{-3} \mathrm{nN} / \mathrm{K}^{2 / 3}$, and $T v^{*} / v=3.9 \times 10^{-2} \mathrm{~K} \mathrm{sec} / \mathrm{nm}$. This compares well to the respective values of $(0.54 \pm$ $0.01) \mathrm{nN}, \quad(1.82 \pm 0.02) \times 10^{-3} \mathrm{nN} / \mathrm{K}^{2 / 3}$, and $(4.5 \pm$ $0.2) \times 10^{-2} \mathrm{~K} \mathrm{sec} / \mathrm{nm}$ extracted from the numerical and experimental work, confirming that the ramped creep regime well describes the motion of the tip. 


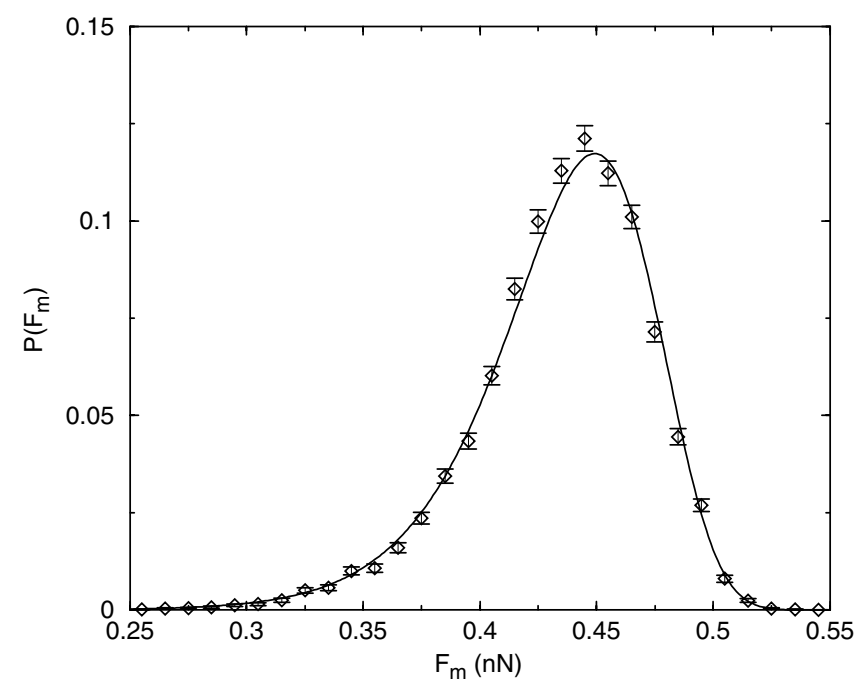

FIG. 3. Normalized distribution, with statistical error bar, of the maxima of the friction force $F_{m}$ at temperature $T=293 \mathrm{~K}$ and velocity $v=25 \mathrm{~nm} / \mathrm{sec}$. The result from the simulation is well reproduced by the theoretical distribution, Eq. (8).

From the distribution function, other properties can be calculated. In particular, a directly accessible experimental quantity is the fluctuation of the maximal force $F_{m}$, the force just before a slip event occurs. Rewriting the bias as $f=1-F_{m} /\left(\tilde{k} R_{c}\right)$ allows us to calculate the distribution of the force maxima. The analytic form is shown in Fig. 3, together with numerical data. The agreement between the numerical results and theoretical prediction is very good. Finally, we note that it is also straightforward to redo the above calculation for the restricted regime in which linear creep takes place. This regime is limited to very small velocities, less than $0.1 \mathrm{~nm} / \mathrm{sec}$ : an expansion of $E(R, x)$ around $\tilde{x}=0$ and $\tilde{x}=a$ yields the condition $v<\left(4 \pi a / \gamma p_{0}^{2}\right)\left(k_{B} T / k\right) \exp \left(-f_{c} U / k_{B} T\right)$, where $f_{c}>0.5$ is the value of the bias at which a linear expansion becomes inappropriate.

To test our results experimentally, it would be particularly valuable to consider an extended range of temperatures and velocities, obtaining not only the dependence of the friction force on those quantities, but the distribution function of such forces as well. The parameters extracted from such comparisons would provide direct, feasible, and simple access to the fundamental description of friction at the atomic scale.

We thank Peter Grütter for useful discussions. This work was supported by the Natural Sciences and Engineering Research Council of Canada, and le Fonds pour la Formation de Chercheurs et l'Aide à la Recherche du Québec.

[1] Handbook of Micro/Nanotribology, edited by B. Bhushan (CRC Press, Boca Raton, Florida, 1999).

[2] B. J. Persson, Sliding Friction (Springer, New York, 1998).
[3] M.C. Mate, G. M. McClelland, R. Erlandsson, and S. Chiang, Phys. Rev. Lett. 59, 1942 (1987).

[4] T. Bouhacina, J.P. Aimé, S. Gauthier, D. Michel, and V. Heroguez, Phys. Rev. B 56, 7694 (1997).

[5] E. Gnecco, R. Bennewitz, T. Gyalog, Ch. Loppacher, M. Bammerlin, E. Meyer, and H.-J. Guntherodt, Phys. Rev. Lett. 84, 1172 (2000).

[6] G. A. Tomlinson, Philos. Mag. 7, 905 (1929).

[7] D. Tománek et al., Europhys. Lett. 15, 887 (1991); T. Gyalog et al., Europhys. Lett. 31, 269 (1995); T. Gyalog and H. Thomas, Z. Phys. B 104, 669 (1997).

[8] J. S. Helman, W. Baltensperger, and J.A. Holyst, Phys. Rev. B 49, 3831 (1994).

[9] H. Hölscher, U. D. Schwarz, and R. Wiesendanger, Europhys. Lett. 36, 19 (1996); Surf. Sci. 375, 395 (1997); O. Zwörner et al., Appl. Phys. A 66, S263 (1998).

[10] J. Colchero, E. Meyer, and O. Marti, in Ref. [1].

[11] F. Heslot, T. Baumberger, B. Perrin, B. Caroli, and C. Caroli, Phys. Rev. E 49, 4973 (1994).

[12] J.D. Gunton and M.C. Yalabik, Phys. Rev. B 18, 6199 (1978).

[13] C. Unger and W. Klein, Phys. Rev. B 29, 2698 (1984).

[14] J. Kurkijarvi, Phys. Rev. B 6, 832 (1972).

[15] L. D. Jackel, W. W. Webb, J. E. Lukens, and S. S. Lei, Phys. Rev. B 9, 4760 (1974).

[16] A. Garg, Phys. Rev. B 51, 15592 (1995).

[17] D. S. Fisher, Phys. Rev. 31, 1396 (1985).

[18] Of course, $\gamma$ is directly related to the coefficient of sliding friction. Calculations of the friction coefficient for adatoms may be found in, e.g., Ref. [2]. The ramped creep regime is valid up to velocities of order $\mathcal{O}\left(F_{c} / M \gamma\right)$, where $F_{c}$ is the constant force in Eq. (1). At this point, the tip hardly feels the barrier potential and hence moves without stick and slip, and the Kramer's activation approach is not valid [9].

[19] Some parameters, such as $\gamma$, can have a weak temperature dependence. Typically, this is negligible, corresponding to a variation of less than $10 \%$ over hundreds of degrees of temperature change. In any event, the scaling forms we propose involve, for example, that temperaturedependent $\gamma$.

[20] M. P. Allen and D. J. Tildesley, Computer Simulations of Liquids (Clarendon, Oxford, 1990).

[21] With the value $U=0.27 \mathrm{eV}$, this corresponds to an elastic constant of value $k=0.93 \mathrm{~N} / \mathrm{m}$.

[22] T. Ala-Nissila and S. C. Ying, Prog. Surf. Sci. 39, 227 (1992).

[23] P. Jung, P. Talkner, and M. Borkocev, Rev. Mod. Phys. 62, 251 (1990).

[24] If the elastic constant between tip and support is small enough that $\langle R\rangle \geq 2 a$, transitions of the tip over more than one lattice spacing can take place. The generic behavior described by Eq. (1) is not modified, except in the academic case of extremely small $\Omega_{k}$.

[25] The Hertz model [2], which neglects activated processes, relates the frictional force to $\left[C(T) F_{L}\right]^{2 / 3}$, where $C$ is inversely proportional to an elastic modulus, and $F_{L}$ is the applied load. This temperature dependence is negligible compared to that obtained herein from the consideration of activated processes, since the temperature dependence of $C$ is small [19]. 